\title{
Physics of String Flux Compactifications
}

\author{
FREDERIK DeneF \\ Institute for Theoretical Physics, University of Leuven, Belgium
}

Michael R. Douglas

Department of Physics and Astronomy, Rutgers University

Institut des Hautes Etudes Scientifiques, Bures-sur-Yvette, France

SHAMIT KACHRU

Department of Physics and SLAC, Stanford University

\begin{abstract}
We provide a qualitative review of flux compactifications of string theory, focusing on broad physical implications and statistical methods of analysis.

\section{CONTENTS}

Introduction

Examples of flux vacua $\ldots \ldots \ldots \ldots \ldots \ldots \ldots$

A toy model . . . . . . . . . . . . . . . . . . . . . . . . . . . . 10

IIa flux vacua . . . . . . . . . . . . . . . . . . . . . 15

More realistic models . . . . . . . . . . . . . . . . . . . 18
\end{abstract}




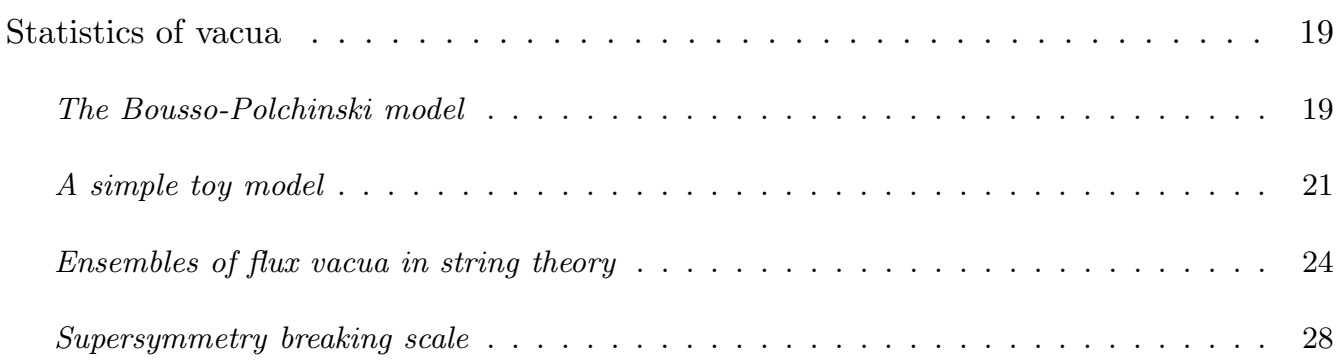

Implications for the testability of string theory . . . . . . . . . . . . . . 34

\section{Introduction}

String theory was first proposed as a candidate theory of quantum gravity in 1974 (1). Over the subsequent years, supersymmetric versions of the theory were developed, and arguments made that they were perturbatively finite (2). The discoveries of anomaly cancellation (3) and of quasi-realistic compactifications of the heterotic string (4) in 1984-85, a period sometimes referred to as the "first superstring revolution," led very rapidly to a broad consensus among particle physicists that superstring theory was a viable contender for a "theory of everything," describing all of fundamental physics.

Now at this point, there were several good reasons to be wary of this claim. One was that there appeared to be many competing theories; besides the different varieties of superstrings, there was eleven-dimensional supergravity. Another was the fact that string theory was defined only as a perturbative expansion, which could only be used directly at weak coupling. Besides the evident fact that the real world includes strongly coupled QCD, various arguments had been made that constructing a completely realistic model would require non-perturbative physics, most notably at the stage of supersymmetry breaking (5).

These doubts were addressed in a rather striking way in the "second super- 
string revolution" of 1994-97, in which it was convincingly argued that all of these theories are limits or aspects of one unified framework, usually now called "string/M theory." The central idea, called duality, is that the strong coupling limit of one of the various string theories, can be equivalent (or dual) to another weakly coupled theory, one of the strings or else M theory, the eleven-dimensional limit (6). Besides providing a variety of nonperturbative definitions for string/M theory, these ideas also led to exact solutions for the effective Lagrangians of a variety of supersymmetric field theories, realizing phenomena such as confinement and chiral symmetry breaking (7). Thus, while much remained to do, it now seemed reasonable to hope that with further theoretical progress, the remaining gaps such as supersymmetry breaking could be addressed.

However, although the particle physics side of the story was moving along nicely, serious gaps remained on other fronts, especially cosmology. The well known cosmological constant problem remained $(8,9)$, as well as the question of whether and how inflation could be described. On a different level, ever since the first studies of string compactification, it had appeared that making any concrete construction required making many arbitrary choices, such as the choice of extra dimensional manifold, a choice of gauge bundle or brane configuration, and so on. Other than the evident constraint that the correct choice should lead to physics which fits the observations, no principles had been proposed, even speculative ones, that would suggest that any of these choices were preferred. Thus it was quite unclear how to get testable predictions from the theory.

A smaller scale version of this problem is that the metric in the extra dimensions, as well as the other data of a solution of string theory, depends on continuous parameters, called moduli. Thus, even having chosen a particular ex- 
tra dimensional manifold, one has many continuous parameters which enter into observable predictions. Again, no particular values of these parameters appeared to be preferred.

At first, one might compare this ambiguity with the choice of coupling constants in a renormalizable field theory. For example, the Standard Model has 19 parameters, and consistency conditions only lead to very weak constraints on these, such as the unitarity bound on the Higgs mass (10). However, the situation here is essentially different - string/M theory has no free parameters, rather each of the parameters of a solution corresponds to a scalar field in four dimensions. If this choice is unconstrained by the equations of motion, this implies that the scalar field is massless. And, massless scalar fields typically (though not always) lead to modifications to the gravitational force law, which are not observed (11). Thus, this is a phenomenological problem, usually called the problem of "moduli stabilization," which must be solved to get realistic models.

Now, having stated the problem, there is a simple argument for why it will generally solve itself in realistic models, as follows. The vacuum structure of a field theory is governed by the effective potential $V_{\text {eff }}(\phi)$, which incorporates all classical and quantum contributions to the potential energy, such as Casimir terms, loop corrections, instantons and the like. These corrections can in principle be computed from the bare Lagrangian, and in almost all cases where this has been done take generic order one values (in units of some fundamental scale) which respect the symmetries of the bare Lagrangian. In particular, this includes the masses of scalar fields. Thus, massless scalar fields never appear in practice, unless they are Goldstone bosons for a continuous symmetry, or unless we can tune parameters (as is done in condensed matter systems to approach critical 
points).

A noteworthy exception is found in supersymmetric field theories, in which nonrenormalization theorems preclude corrections to the superpotential to all orders in perturbation theory. While some theories admit non-perturbative corrections, others do not, and in those theories moduli are natural. However, a realistic model must break supersymmetry at some scale $M_{\text {susy }} \geq 1 \mathrm{TeV}$, and below this scale the previous argument applies. Thus, the moduli will ultimately gain masses of order $m \sim c M_{\text {susy }}$, where $c$ is often a small number of the order $M_{\text {susy }} / M_{P}$.

This solution turns out to be problematic $(12,13)$, as it leads to the so-called Polonyi problem (14), wherein the light moduli fields carry too much energy in the early universe, leading to overclosure. Thus, one is led to look for other mechanisms which could give larger masses to moduli. A particularly simple mechanism, which we discuss in some detail in section 2 , is to postulate a background generalized magnetic field in the extra dimensions, usually called "flux." The energy of such a field will depend on the moduli, and provides a new contribution to the effective potential. Its scale is set by the unit of quantization (a fundamental scale such as the string scale) and the inverse size of the extra dimensions. This is typically far higher than $M_{\text {susy }}$ and solves the problem.

But, there is an unexpected side effect of this mechanism. On a qualitative level, it works for fairly generic nonzero choices of flux. Supposing that each flux can take of order 10 values, then one finds of order $10^{K}$ distinct solutions, where $K$ is the number of distinct topological types of flux (usually a Betti number). And, the compactification manifolds used in string theory, say the Calabi-Yau manifolds, typically have $K \sim 30-200$. Thus, one finds a large vacuum multiplicity. Furthermore, physical predictions depend on this choice, 
both directly and because the values of the stabilized moduli depend on the flux.

As one might imagine and as we discuss below, this vacuum multiplicity makes it rather complicated to propose definitive tests of the theory. Philosophically, it seems very much at odds with the idea that a fundamental theory should be simple and unique. Now it is hard to know how much weight to put on such considerations, which do not in themselves bear on the truth or falsity of the theory as a description of nature, but certainly one should ask for more evidence before accepting this picture.

In a seemingly unrelated development, starting in the late 1990's, convincing evidence has accumulated for a non-zero dark energy in our universe, from the accelerated expansion as measured by observations of supernova, in precision measurements of the CMB, and from other sources (15). Although not absolutely proven, the simplest model for this dark energy is a positive cosmological constant, of order $\Lambda=(0.71 \pm 0.01) \Omega$, where $\Omega$ is the critical density.

This observation brought the cosmological constant problem to the fore of fundamental physics. As long as the data was consistent with $\Lambda \sim 0$, the simplest hypothesis was that the correct theory would contain some mechanism which adjusted $\Lambda$ to zero. Many proposals along these lines have been made, and although none is generally accepted, nor is there any proof that this is impossible. On the other hand, the existing proposals generally do not fit well within string theory, and it had been widely felt that a proper solution would require some essentially new idea.

On the other hand, the problem of fitting a specific non-zero $\Lambda$ looks rather different, indeed the particular choice of value seems to be rather arbitrary. Its one evident property is that it is of the same order as the matter density, but 
only at the present epoch (the coincidence problem). This led to the study of proposals such as quintessence in which the dark energy is time dependent; we will only say here that this proposal has the advantage of being directly testable, and so far the evidence seems to be coming down on the other side, for a fixed cosmological constant.

Now, there was one previous proposal for a mechanism which could explain a small non-zero cosmological constant, due to Banks (16), Linde (17) and particularly Weinberg (18). This was the idea that the underlying theory might manifest a large number of distinct vacua, which are stable at least on cosmological time scales, and realize different values of the cosmological term $\Lambda$. When Weinberg was writing, the direct observational bounds on $\Lambda$ placed a bound on its value which had just excluded the range of $\Lambda$ s which are too large to allow successful galaxy formation. Weinberg advocated the point of view that given that $\Lambda$ is absurdly small compared to fundamental scales in Nature (indeed, at the time he wrote, there was only a tiny upper bound on its value), one should postulate that the distribution of $\Lambda$ in the different vacua is uniform in the observationally allowed range, that compatible with galaxy formation. It then follows statistically that most vacua which are compatible with observations would lie within a decade or two of the maximal allowed value. Thus, Weinberg predicted that the eventual observed $\Lambda$ would be comparable to the maximal value compatible with galaxy formation. This has proved true.

An important ingredient in this argument is that the fundamental underying theory should admit many vacua. While from the beginning, the choices present in string compactification made it clear that the theory has many distinct solutions, the number required by Weinberg's argument (roughly $10^{120}$ ) was 
far larger than any reliable approximation of the number of metastable vacua. Though some estimates of very large numbers of constructions were made by counting distinct soluble points (lattice constructions or orbifold models) in continuous moduli spaces of vacua (19), it was widely believed and still seems very likely to be true that these many constructions would collapse into a few after supersymmetry breaking, since they were simply distinct points on the same supersymmetric moduli space. The number of discretely differing solutions seemed far smaller: for instance, while Calabi-Yau spaces are far from unique, the number of distinct choices after more than twenty years of systematically searching for constructions still numbers in the thousands. This fact, together with the inability to exhibit reliable metastable solutions after supersymmetry breaking, largely discouraged speculation on this topic.

The duality revolution, however, brought many new ingredients of string theory to the fore. One of these was the generalized gauge fields which couple to D-branes. Each of these gauge fields come with an associated field strength, whose flux can thread the extra dimensions. It was realized in (20) that in compact manifolds with sufficiently complicated topology, the number of flux choices allowed by the known constraints could easily exceed $10^{120}$. This provided a large, discretely varying collection of models, which could realize different values of the cosmological term. While this model neglected many aspects of the physics, in particular simply freezing the moduli of the internal space by hand, it made it very plausible that string theory could contain the large set of vacua required by Weinberg's argument.

In light of this combination of theoretical and observational considerations, it has become very interesting to find concrete and computable models of string 
compactification in which the moduli problem is solved, and in which distinct choices of flux provide a large collection of vacua. Starting around 2000, this problem received a great deal of attention (some representative works being (21, $22,23,24,25,26)$ ), with a milestone being the KKLT proposal (27) for a class of IIb compactifications in which all moduli are stabilized, by a combination of fluxes and non-perturbative effects, while supersymmetry is broken with a small positive cosmological constant. Subsequent work has proposed more and more concrete realizations of this proposal $(28,29,30)$, as well as alternate constructions using the other string theories and $\mathrm{M}$ theory, or relying on different hypotheses for the scales and dominant terms in the effective potential. One can (heuristically) envision these potentials as taking values over a large configuration space, which has been called the "string landscape" (31). A central question in recent years has been to characterize the landscape, and find dynamical mechanisms for populating and selecting among the vacua within it.

A full discussion of these constructions very quickly becomes technical, and we refer to reviews such as $(32,33,34,35,36)$ for the details. However, we will outline one representative set of constructions in the next section, to illustrate the ideas.

In the rest of the review, we begin to address the questions of testability raised by the landscape. In section 3, we discuss formal results for the distribution of vacua and their observable properties, while in section 4 , we survey the various approaches for getting concrete and testable predictions from the framework.

Many related topics had to be omitted for reasons of space, such as inflation in string theory. We were also limited to citing only major reviews and a few influential and/or particularly recent papers, and again direct readers to (32, 33 , 34, 35, 36) for a more complete bibliography. 


\section{Examples of flux vacua}

In this section, we describe in more detail the ingredients that enter in string flux compactifications. We first give a simple toy model that captures much of the relevant physics. In $\S 2.2$, we extend this to a full discussion of a simple class of flux vacua of IIa string theory. In $\S 2.3$, we briefly describe other ingredients that enter in making quasi-realistic models.

\section{$2.1 \quad$ A toy model}

The essential point will be that fluxes and branes present in 10d string theory provide natural ingredients for stabilizing the moduli of a compactification. However the basic ideas are independent of dimension and the simplest illustration involves 6d Einstein-Maxwell theory. We imagine, therefore, a 6d theory whose dynamical degrees of freedom include the metric $g_{M N}$ and an abelian Maxwell field $F_{M N}$. The Lagrangian takes the form

$$
L=\int d^{6} x \sqrt{-g}\left(M_{6}^{4} \mathcal{R}-M_{6}^{2}|F|^{2}\right)
$$

where $M_{6}$ is a fundamental unit of mass (the $6 \mathrm{~d}$ Planck scale).

To define a compactification of this $6 \mathrm{~d}$ theory to four dimensions, one must choose a compact $2 \mathrm{~d}$ internal space. The topologies of $2 \mathrm{~d}$ manifolds without boundary are classified by the genus, i.e., the possible spaces $M_{g}$ are (hollow) donuts with $g=0,1,2, \cdots$ holes. $g=0$ is the sphere, $g=1$ is the two-torus, and so forth.

Suppose we compactify the theory on the manifold $M_{g}$ of genus $g$ and total volume $R^{2}$. As an ansatz for the $6 \mathrm{~d}$ metric, we could take

$$
d s^{2}=g_{\mu \nu} d x^{\mu} d x^{\nu}+R^{2} \tilde{g}_{m n} d y^{m} d y^{n}
$$


with $\tilde{g}$ a metric of unit volume on $M_{g}$. Our focus here will be the dynamics of the "modulus field" $R(x)$ which enters into the ansatz Eq. (2).

We can expand the resulting $4 \mathrm{~d}$ effective Lagrangian in powers of derivatives, as is standard in effective field theory. The leading terms arising from the gravitational sector are

$$
M_{6}^{4} R^{2} \int d^{4} x \sqrt{-g}\left(\left[\int d^{2} y \sqrt{\tilde{g}} \mathcal{R}_{2}\right]+R^{2} \mathcal{R}_{4}\right)+\cdots
$$

Here, $\mathcal{R}_{2}$ is the curvature of $\tilde{g}$ and $\mathcal{R}_{4}$ is the curvature of the $4 \mathrm{~d}$ spacetime metric $g$. The $\cdots$ includes both gradients of $R$ and terms involving the $4 \mathrm{~d}$ gauge field.

There are two important points about the action as written above. Firstly, defining $M_{4}^{2}=M_{6}^{4} R^{2}$ as the 4 d Planck scale, we see the action is not in Einstein frame - the kinetic terms of $R$ and the graviton are mixed. Secondly, the first term in brackets above is in fact a topological invariant, $\chi\left(M_{g}\right)$. For a surface of genus $g, \chi=2-2 g$. We therefore find a scalar potential for the modulus field unless $g=1$.

To go to 4d Einstein frame, we should redefine the 4d metric: $g \rightarrow h=R^{2} g$. Then $\sqrt{h} \mathcal{R}_{h}=R^{2} \sqrt{g} \mathcal{R}_{g}$, and we find a $4 \mathrm{~d}$ Lagrangian

$$
M_{4}^{2} \int d^{4} x \sqrt{-h}\left(\mathcal{R}_{h}-V(R)\right)
$$

The potential $V(R)$ is given by

$$
V(R) \sim(2 g-2) \frac{1}{R^{4}}
$$

The $R$ dependence just follows from the Weyl rescaling above.

We have learned an interesting lesson. In the absence of background Maxwell fields, of the infinite family of compactification topologies parametrized by $g$, precisely one choice yields a static solution. The choice $g=1$, the two-torus, 
yields a vanishing potential and a $4 \mathrm{~d}$ flat-space solution. The positive curvature $g=0$ surface, the two-sphere $S^{2}$, naively has a negative potential $\sim-\frac{1}{R^{4}}$, yielding runaway to $R \rightarrow 0$ (where the regime of trustworthiness of our analysis breaks down). The negative curvature options, $g>1$, instead yield positive potentials, which vanish as $R \rightarrow \infty$ - they spontaneously "decompactify" back to the $6 \mathrm{~d}$ flat space solution.

All of these features of our toy model have analogues in full-fledged string compactification. The $T^{2}$ here, which is Ricci-flat, is analogous to the Calabi-Yau solutions which have been intensely studied by string theorists. One difference is that while $T^{2}$ is a unique 2-manifold, there are at least thousands of topologically distinct Calabi-Yau threefolds, each leading to a class of compactifications. If our $6 \mathrm{~d}$ theory were promoted to a supersymmetric Einstein-Maxwell theory, this 4d flat solution would have unbroken supersymmetry at the KK scale (which partially explains the fascination that string theorists have had with the analog Calabi-Yau solutions). Notice that the modulus field $R$ survives "unfixed" in the $4 \mathrm{~d}$ effective theory - it appears to $4 \mathrm{~d}$ physicists as a massless scalar field. In fact the $T^{2}$ has an additional modulus corresponding to its shape; the CalabiYau spaces also manifest both volume moduli and "shape" (complex structure) moduli.

The $S^{2}$ case, with positive curvature, mirrors the Einstein manifolds used in Freund-Rubin compactification (37) - more on this momentarily. The negative curvature manifolds (here, the most common topologies) are just now being examined as candidate string compactifications (38).

2.1.1 InCLUding FLuXes AND BRANes Let us do this step by step, beginning by turning on the Maxwell field. To find $4 \mathrm{~d}$ vacuum solutions with maximal 
symmetry, we should only allow the gauge field configuration to be nontrivial on $M_{g}$. The most obvious possibility is to thread $M_{g}$ with some number of units of magnetic flux of the gauge field

$$
\int_{M_{g}} F=n
$$

Like a magnetic flux threading a solenoid, this flux through $M_{g}$ carries positive energy density. This makes an additional contribution to the $4 \mathrm{~d}$ effective potential as a function of $R$. Naively, it scales like $\frac{1}{R^{2}}$ - a factor of $R^{2}$ from the determinant of the metric, and two factors of $1 / R^{2}$ from the metric factors contracting the indices on $F_{m n}$. However, upon rescaling to reach 4 d Einstein frame, one multiplies by an additional $1 / R^{4}$, so the full potential takes the schematic form

$$
V(R) \sim(2 g-2) \frac{1}{R^{4}}+\frac{n^{2}}{R^{6}}
$$

In the presence of $n$ units of magnetic flux, the status of the different compactification topologies changes.

Previously, the $g=0 S^{2}$ compactification yielded solutions that run to (uncontrolled) small values of $R$. However, it is easy to see that with $n$ units of flux, the positive flux energy can balance the negative curvature contribution at a finite values of $R$, which grows with $n$. Therefore, for large $n$, one obtains reliable flux vacua from a large $S^{2}$ with $n$ units of flux piercing it. These are the Freund-Rubin solutions.

For $g=1$, where previously there was a moduli space of solutions, the flux causes a runaway to large $R$. Finally, for $g>1$, since both terms in the potential are positive and vanish at large $R$, there is also a runaway.

The same behavior obtains in the full string theory. The Freund-Rubin solutions also exist there, and play a crucial role in e.g. the AdS/CFT correspondence. 
There are indeed no solutions of string theory which start from (supersymmetric) Calabi-Yau compactification and incorporate only flux - additional ingredients are needed (which we will come to next). Finally, the negative curvature spaces also require additional ingredients to avoid runaway.

Next, we add branes, dynamical, fluctuating lower-dimensional objects which can carry gauge and matter fields. A common example is the Dirichlet (or D) brane. These have positive tension and thus make positive contributions to $V(R)$. String theories also include fixed, non-dynamical objects, the so-called orientifold planes or O-planes, with negative tension.

We now modify our toy model to include some fixed $\mathcal{O}(1)$ number $m$ of O3planes in the background, at points on $M_{g}$. Taking into account Weyl rescaling, their contribution to the $4 \mathrm{~d}$ effective potential is

$$
\delta V_{O 3}=-m \frac{1}{R^{4}}
$$

We see that after inclusion of these objects, the full effective potential of our toy model takes the general form

$$
V(R)=(2 g-2) \frac{1}{R^{4}}-m \frac{1}{R^{4}}+n^{2} \frac{1}{R^{6}} .
$$

The physics of Eq. (9) captures many of the qualitatively important facts in flux compactification (though the identical scaling of the O-plane and curvature contributions will not hold in the string analogues). Of course it is an oversimplification: in real constructions there are many further constraints, determining the number of branes, planes and so forth.

Including both branes and fluxes, we see that if models with suitable $m$ are available, (a) the modulus $R$ of the $T^{2}$ compactification can be fixed by inclusion of O3 planes, and (b) the negative curvature models may now admit critical 
points of their potential at large $R$ (for string theory examples, see (39)).

The vacua analogous to type (a) will be our focus in much of this review. These are Calabi-Yau models with an underlying $\mathcal{N}=2$ supersymmetry, broken to $\mathcal{N}=1$ by the branes and O-planes. Other ingredients, such as non-perturbative physics, are required to stabilize all moduli and break supersymmetry, as described in (32).

In many of these models, the scale of supersymmetry breaking can be very low compared to the KK scale. Thus these models are a logical place to search for string extensions of the MSSM, which include its coupling to quantum gravity.

\subsection{IIa flux vacua}

The simplest full constructions of string vacua along these lines use IIa string theory $(40,41,42)$. This theory contains $\mathrm{p}$-form RR field strengths $F_{p}$ with $p=$ $0,2,4,6,8$, and an NS field strength $H_{3}$. Practically, this means that in addition to the possible fluxes threading nontrivial $0,2,3,4$ and 6 cycles of the 6 -dimensional compactification manifold, the theory contains D0,2,4,6,8 branes and NS 5 branes. Along with the D-branes, there can be O-planes of each even dimension $<10$. Any of these objects can form part of a solution with $4 \mathrm{~d}$ rotational symmetry if they "fill" $4 \mathrm{~d}$ space-time, and thus "wrap" a $p-3$ dimensional cycle in the compactification manifold.

We can analyze the resulting effective potential in close analogy to the one for our toy model. Let us imagine compactification on a Ricci-flat 6-manifold $M$ (say a Calabi-Yau) with $b_{2}=b_{4}$ topologically distinct 4 -cycles, $b_{3}$ distinct 3 -cycles, no 1-cycles or 5-cycles, and volume $R^{6}$.

Unlike our toy model, in string theory there is no predetermined dimensionless 
coupling; the $10 \mathrm{~d}$ coupling $g_{s}$ is determined by the dilaton field $\phi$ as $g_{s}=e^{\phi}$. A minimal discussion therefore focuses on the dynamics of the volume modulus $R$ and $\phi$, and we shall do that below, sketching the elaboration to include other moduli at the end. In a full discussion, all moduli must be fixed.

Because the manifold is Ricci-flat, there is no contribution to the potential from the $10 \mathrm{~d}$ curvature term. Thus, to prevent a runaway to large $R$, we will need to include O-planes. Given the assumed topology of $M$, these must be O6-planes wrapping 3-cycles.

We will also need to know the $g_{s}$ scaling of the various sources of energy in ten dimensions. In $10 \mathrm{~d}$ string frame, the Einstein term scales like $e^{-2 \phi}$, while the D-brane/O-plane tensions scale like $e^{-\phi}$. In addition, the RR $|F|^{2}$ terms are independent of $g_{s}$ (in the convention where the RR flux quantization is $g$ independent), while the NS $|H|^{2}$ arises at order $e^{-2 \phi}$.

We can now write a schematic effective potential for $R$ and $e^{\phi}$, given any particular choice of ingredients. One natural class of compactifications involves $N$ unit of RR $F_{4}$ flux, $\mathcal{O}(1)$ units of $F_{0}$ flux and $H_{3}$ flux, and some fixed $\mathcal{O}(1)$ number of $\mathrm{O} 6$ planes. The resulting potential is

$$
V=N^{2} \frac{e^{4 \phi}}{R^{14}}+\frac{e^{2 \phi}}{R^{12}}-\frac{e^{3 \phi}}{R^{9}}+\frac{e^{4 \phi}}{R^{6}}
$$

One sees that this potential admits vacua with $R \sim N^{1 / 4}$ and and $g_{s}=e^{\phi} \sim$ $N^{-3 / 4}$. So by analogy with the Freund-Rubin toy model, this theory has vacua with large volume (and weak coupling) if one turns on a large number of flux quanta. An important distinction between this model and the Freund-Rubin vacua is that the size of the compact space $M$ is parametrically smaller, in this leading approximation, than the curvature radius set by the leading estimate for the $4 \mathrm{~d}$ cosmological term. So these models are indeed models of $4 \mathrm{~d}$ matter 
coupled to quantum gravity, over some range of scales.

This discussion has clearly oversimplified the complex problem of stabilizing Calabi-Yau moduli. However, the basic construction above does in fact yield models with all moduli stabilized in the full string theory. The simplest example (40) uses the Calabi-Yau orbifold $T^{6} / Z_{3}^{2}$. This space is a quotient of the six-torus by the action of two discrete symmetry groups. We start with the torus described by complex coordinates $z_{i}=x_{i}+i y_{i}, i=1, . ., 3$, subject to identifications

$$
z_{i} \sim z_{i}+1 \sim z_{1}+\alpha
$$

where $\alpha=e^{i \pi / 3}$. This torus has a $Z_{3}$ symmetry $T$ under which

$$
T:\left(z_{1}, z_{2}, z_{3}\right) \rightarrow\left(\alpha^{2} z_{1}, \alpha^{2} z_{2}, \alpha^{2} z_{3}\right)
$$

This action has a total of $3 \times 3 \times 3=27$ fixed points on the torus. While these result in conical singularities, these are allowed in perturbative string theory (43). One can reduce this to 9 singularities by quotienting by another, freely acting $Z_{3}$ symmetry $Q:\left(z_{1}, z_{2}, z_{3}\right) \rightarrow\left(\alpha^{2} z_{1}+\frac{1+\alpha}{3}, \alpha^{4} z_{2}+\frac{1+\alpha}{3}, z_{3}+\frac{1+\alpha}{3}\right)$.

Orbifolds by discrete subgroups of $S U(3)$ give rise to (in general singular) Calabi-Yau manifolds, so our construction thus far gives an $\mathcal{N}=2$ type IIa compactification on a Calabi-Yau space. To break the supersymmetry to $\mathcal{N}=1$, we now orientifold by the simultaneous action of the $Z_{2}$ involution

$$
\sigma: z_{i} \rightarrow-\overline{z_{i}}
$$

with worldsheet parity reversal. The action of $\sigma$ gives rise to a fixed 3-plane $z_{i}=0$, which is wrapped by a space-filling O6 plane.

The model we have described has 12 volume moduli, whose origin is as follows: three of them simply rescale the volumes of the three two-tori involved in the 
construction. The other 9, one per singularity, are associated to "exceptional cycles" introduced by resolving the $C^{3} / Z_{3}$ fixed points. On the other hand, it has no complex structure moduli (it is "rigid"), because the $Z_{3}$ symmetries only arise for a unique shape of the covering torus, and because resolving the singularities introduces only volume moduli.

It is now straightforward to add ingredients which give rise to a potential analogous to that in Eq. (10). We already have an O6 plane; one can thread the complementary three-cycle with $H_{3}$ flux, and turn on $F_{0}$ flux and $F_{4}$ flux through the 4-cycles transverse to the $T^{2} \mathrm{~S}$ of the original $\left(T^{2}\right)^{3}$. The one novelty is the existence of the exceptional cycles, but these can be stabilized at large volume by four-form flux as well. The details can be found in (40); the result is a model in which the leading effective potential stabilizes the moduli at weak string coupling and large volume, just as suggested by Eq. (10). It should be admitted that this analysis is not an absolute proof of existence; for possible subtleties see (44).

\subsection{More realistic models}

We have focused here on construction of models with computable moduli potentials. To make contact with observed physics, we also need a sector giving rise to (a supersymmetric extension of) the Standard Model, and perhaps a sector which is responsible for supersymmetry breaking and its transmission to the Standard Model. There has been significant progress on constructing flux vacua which incorporate all of these elements.

One popular method for constructing Standard-like models in string theory is to use intersecting D-branes, for example D6 branes in IIa theory. Stacks of $N$ parallel branes manifest an $S U(N)$ gauge theory, while intersections of D6-branes 
at points in the extra dimensions localize chiral matter multiplets (45). These ingredients allow "engineering" the Standard Model, and indeed fairly general $\mathcal{N}=1$ gauge theories. For the state of the art, see (46).

One can also use this to construct models of dynamical supersymmetry breaking. It has recently become clear that even the simplest non-chiral gauge theories have supersymmetry breaking vacua (47), and one can very simply engineer these constructions on D6 branes (48). Another option is to arrange for the flux potential itself to provide the supersymmetry breaking, as we will discuss in the next section. Thus it is quite plausible that the class of models we described above includes the supersymmetric Standard Model and its various extensions, with supersymmetry breaking transmitted via gravity or gauge interactions.

\section{Statistics of vacua}

We now supply a brief introduction to and survey of this topic, referring to (32,49) for more extensive reviews.

\subsection{The Bousso-Polchinski model}

As pointed out in (20), the freedom one has to turn on various independent flux quanta in string theory compactifications leads to ensembles of vacua with a variety or "discretuum" of low energy effective parameters. This leads to a need for statistical analysis of the resulting vacuum distributions (50).

This is true in particular for the cosmological constant, implying naturally the existence of string vacua with exceedingly small effective four dimensional cosmological constants, such as our own, without the need to invoke any (so far elusive) dynamical mechanism to almost-cancel the vacuum energy. 
To see how this comes about, consider the (classical) potential induced by a flux $F$ characterized by flux quanta $N^{i} \in \mathbb{Z}, i=1, \ldots, K$, given by

$$
V_{N}(\phi)=V_{0}(\phi)+\int_{X}\|F\|^{2}=V_{0}(\phi)+\sum_{i, j} g_{i j}(\phi) N^{i} N^{j}
$$

where $\phi$ denotes the moduli of the compactification manifold $X$ and $g_{i j}(\phi)$ is some positive definite effective metric on the moduli space. The number of fluxes $K$ is typically given by the number of homologically inequivalent closed cycles of some fixed dimension in $X$, which for the known examples of six dimensional Calabi-Yau manifolds is typically of order a few hundred. The bare potential $V_{0}(\phi)$ is taken to be negative. In string compactifications it could e.g. come from orientifold plane contributions, and in this context $V_{0}$ (as well as $g_{i j}$ ) will be of order of some fundamental scale such as the string or Kaluza-Klein scale.

Each vacuum of this model is characterized by a choice of flux vector $N$ together with a minimum $\phi_{*}$ of $V_{N}(\phi)$. Finding these critical points explicitly is typically impossible, so to make progress one has to use indirect statistical methods, as we will discuss further on. However, before plunging in to this full, coupled problem, let us, following (20), first simply freeze the moduli at some fixed value $\phi=\phi_{0}$ and ignore their dynamics altogether. In that case it is easy to compute the distribution of cosmological constant values: the number of vacua with cosmological constant $\Lambda=V_{N}\left(\phi_{0}\right)$ less than $\Lambda_{*}$ is then simply given by the number of flux lattice points in a sphere of radius squared $R^{2}=\left|V_{0}\right|+\Lambda_{*}$, measured in the $g_{i j}$ metric. When $R$ is sufficiently large, this is well-estimated by the volume of this ball, i.e. $\operatorname{Vol}_{K}(R)=\frac{\pi^{K / 2}}{\Gamma(1+K / 2)} \frac{R^{K}}{\sqrt{\operatorname{det} g}}$, leading to a $\Lambda$-distribution

$$
d N_{\operatorname{vac}}(\Lambda)=\frac{\pi^{K / 2}}{\Gamma(K / 2)} \frac{\left(\left|V_{0}\right|+\Lambda\right)^{\frac{K}{2}-1}}{\sqrt{\operatorname{det} g}} d \Lambda \approx\left(\frac{2 \pi e\left(\left|V_{0}\right|+\Lambda\right)}{\mu^{4}}\right)^{K / 2} \frac{d \Lambda}{\left|V_{0}\right|+\Lambda}
$$

where $\mu^{4}:=(\operatorname{det} g)^{1 / K}$ can be interpreted as the mass scale of the flux part of 
the potential and we assumed large $K$ and used Stirling's formula to get the last approximate expression. Note that in particular at $\Lambda=0$, for say $\left|V_{0}\right| / \mu^{4} \sim$ $\mathcal{O}(10)$, we get a vacuum density $d N_{\text {vac }} \sim 10^{K / 2} d \Lambda /\left|V_{0}\right|$. Hence for $K$ a few hundred, there will be exponentially many vacua with $\Lambda$ in the observed range $\Lambda \sim 10^{-120} M_{p}^{4}$, even if all fundamental scales setting the parameters of the potential are of order $M_{p}^{4}$.

In such a model, there is no need to postulate either anomalously large or small numbers, or an unknown dynamical mechanism, to obtain vacua with a small cosmological constant. Combined with a cosmological mechanism generating all possible vacua (such as eternal inflation) and Weinberg's argument as discussed in the introduction, we have a candidate solution to the cosmological constant problem within string theory.

Of course, the Bousso-Polchinski model is only a crude approximation to actual flux vacua of string theory; in particular freezing the moduli at arbitrary values is a major oversimplification. However, more general and more refined analyzes of low energy effective parameter distributions of actual string flux vacua taking moduli dynamics into account, initiated in (50) and further developed in $(51,52$, 53), confirmed the general qualitative features of the model we just discussed. We now turn to an overview of these studies.

\section{$3.2 \quad$ A simple toy model}

Let us demonstrate the basic ideas behind these techniques by considering the following ensemble of effective potentials:

$$
V_{N, M}(\phi)=N \phi+M \frac{\phi^{2}}{2}, \quad N, M \in \mathbb{Z}, \quad N^{2}+M^{2} \leq L .
$$


Here the $(N, M)$ (crudely) model fluxes, while $\phi$ models a modulus field. To get a large number of vacua, we take $L$ to be very large. Finding the critical points is trivial for this ensemble: they are given by $\phi_{*}=-N / M$, and are stable iff $M>0$. To find the distribution of vacua over $\phi$-space in the large $L$ limit, we could in principle start from these explicit solutions. However, a more elegant and powerful method, which is also applicable to cases for which explicit solutions cannot be found, and to ensembles of actual string theory flux vacua, goes as follows.

The number of vacua $\phi_{*}$ in an interval $I$ is given by

$$
\mathcal{N}_{\mathrm{vac}}(I)=\sum_{N, M} \int_{I} d \phi \delta\left(V^{\prime}(\phi)\right)\left|V^{\prime \prime}(\phi)\right| \theta\left(V^{\prime \prime}(\phi)\right),
$$

where $\theta(x):=1$ if $x>0, \theta(x):=0$ if $x<0$. The integrand $\delta\left(V^{\prime}\right)\left|V^{\prime \prime}\right|$ gives a contribution +1 for each critical point in $I$, while $\theta\left(V^{\prime \prime}\right)$ restricts to actual minima.

Now in the large $L$ limit, we can approximate the sum over $(N, M)$ by an integral, and write

$$
\mathcal{N}_{\mathrm{vac}}(I) \approx \int_{I} d \phi \rho(\phi), \quad \rho(\phi):=\int d N d M \delta\left(V^{\prime}(\phi)\right) V^{\prime \prime}(\phi) \theta\left(V^{\prime \prime}(\phi)\right),
$$

where $\rho(\phi)$ can be interpreted as a vacuum number density on moduli space. To evaluate the integral over $(N, M)$ at a given fixed $\phi$, it is convenient to make the following linear change of variables $(N, M) \rightarrow\left(v^{\prime}, v^{\prime \prime}\right)$ :

$$
v^{\prime}:=V^{\prime}(\phi)=N+M \phi, \quad v^{\prime \prime}:=V^{\prime \prime}(\phi)=M .
$$

This change of variables has Jacobian $=1$, and the integration domain in the new variables is $L \geq N^{2}+M^{2}=\left(v^{\prime}-v^{\prime \prime} \phi\right)^{2}+\left(v^{\prime \prime}\right)^{2}$. The integral is now trivially evaluated, yielding the distribution

$$
\rho(\phi)=\frac{L}{2} \frac{1}{1+\phi^{2}}
$$


Note that this integrates to at total number of vacua $\mathcal{N}_{\text {vac }}(\mathbb{R}) \approx \pi L / 2$ - this is as it should, since the total number of pairs $(N, M)$ in the ensemble is approximated by the volume $\pi L$ of the region $N^{2}+M^{2} \leq L$, each $(N, M)$ leads to a unique critical point, and half of those are minima. The density Eq. (20) confirms the intuitive expectation that "most" vacua in this ensemble will be at order 1 values of $\phi$, but makes this far more precise.

Let us next consider a more general ensemble of the form

$$
V_{N, M}(\phi)=N f(\phi)+M g(\phi)
$$

where $f$ and $g$ are arbitrary functions. Now it is no longer possible to proceed by finding explicit solutions - even finding a single explicit solution will typically be out of reach even for simple choices of $f$ and $g$. On the other hand, the previous computation is straightforwardly extended to this case, for general $f$ and $g$, resulting in a vacuum number density

$$
\rho(\phi) d \phi=\frac{L}{2} \frac{\left|f^{\prime} g^{\prime \prime}-f^{\prime \prime} g^{\prime}\right|}{\left(f^{\prime}\right)^{2}+\left(g^{\prime}\right)^{2}} d \phi=\frac{L}{2} \operatorname{sign}\left(\frac{g^{\prime}}{f^{\prime}}\right)^{\prime} d \arctan \left(\frac{g^{\prime}}{f^{\prime}}\right) .
$$

This illustrates the power of statistical methods over explicit constructions.

An interesting special case, which has an important counterpart in actual string flux vacua, is obtained by setting $f(\phi)=\phi, g(x)=\phi \log \phi-\phi, \phi>0$. Potentials with similar structure appear naturally in string theory, as we will discuss in more detail below. The corresponding vacuum density is

$$
\rho(\phi) d \phi=\frac{L}{2} \frac{1}{\left(1+\log ^{2} \phi\right)} \frac{d \phi}{\phi}=\frac{L}{2} d \arctan \log \phi .
$$

Note that this distribution is approximately scale invariant, and thus naturally allows hierarchically small (and large) vacuum values of $\phi$ and therefore $V(\phi)$. For this particular ensemble, we can also see this directly as the critical points can again be found explicitly, namely $\phi_{*}=e^{-N / M}$. 


\subsection{Ensembles of flux vacua in string theory}

The most studied and best understood ensemble of flux vacua is the IIb ensemble, which arises by allowing two different kinds of fluxes (RR and NSNS) to be turned on through nontrivial 3-cycles of a Calabi-Yau compactification space $X$. There are two kinds of geometric moduli, arising from complex structure (shape) and Kähler (size) deformations of $X$. Besides these, there is a universal modulus $\tau=C_{0}+i / g_{s}$, where $C_{0}$ is the universal axionic scalar of the IIb theory and $g_{s}$ is the string coupling constant. The complex structure moduli and $\tau$ appear nontrivially in the potential induced by the fluxes and as a result are generically stabilized at tree level. The Kähler moduli on the other hand do not but can under certain conditions be stabilized by quantum effects (27). We will assume this is the case and just freeze them in the following.

The potential for this ensemble has the standard $\mathcal{N}=1$ supergravity form

$$
V_{N}(z, \bar{z})=e^{K}\left(g^{a \bar{b}} D_{a} W_{N} \overline{D_{b} W_{N}}-3\left|W_{N}\right|^{2}\right)
$$

where $g_{a \bar{b}}=\partial_{a} \bar{\partial}_{\bar{b}} K, D_{a}=\partial_{a}+\partial_{a} K$ and

$$
W_{N}(z)=\sum_{i=1}^{K} N^{i} \Pi_{i}(z), \quad K(z, \bar{z})=-\ln \left(Q^{i j} \Pi_{i}(z) \overline{\Pi_{j}(z)}\right) .
$$

Here $z^{a}$ denotes the complex structure coordinates together with $\tau, Q^{i j}$ is a known constant $K \times K$ matrix and $\Pi_{i}(z)$ are certain complicated but in principle computable holomorphic functions (the periods), which depend on the CalabiYau $X$ at hand. Note that the potential is quadratic in the $N_{i}$, as in the BoussoPolchinski model. The number of flux quanta is $K=2 b_{3}(X)$, where $b_{3}(X)$ is the third Betti number of $X$, i.e. the number of homologically independent 3-cycles. For the quintic Calabi-Yau, which can be described as the zero locus of any homogeneous degree 5 polynomial in $\mathbb{C}^{5}$ with points related by overall complex 
rescaling identified, one has $b_{3}=204$.

Finally, there is a tadpole cancellation constraint on the fluxes, of the form

$$
\frac{1}{2} Q^{i j} N_{i} N_{j} \leq L
$$

where $L$ depends again on the compactification data but typically ranges from $\mathcal{O}(10)$ to $\mathcal{O}(1000)$

Supersymmetric vacua of this model are given by solutions of $D_{a} W_{N}(z)=$ 0. Note that counting these amounts to a direct generalization of the counting problem of our toy model, with the periods $\Pi_{i}(z)$ generalizing $f(\phi)$ and $g(\phi)$. And indeed it turns out to be possible to derive approximate distributions at large $L$ using essentially the same ideas $(51,52)$. The resulting distribution 1 of vacua over moduli space turns out to be surprisingly simple:

$$
d N_{\mathrm{vac}}(z)=\frac{(2 \pi L)^{K / 2}}{\left(\frac{K}{2}\right) ! \pi^{K / 4}} \operatorname{det}(R(z)+\omega(z) \mathbf{1}),
$$

where $R(z)=\frac{i}{2} R_{b c \bar{d}}^{a} d z^{c} \wedge d \bar{z}^{\bar{d}}$ is the curvature form of the metric $g_{a \bar{b}}$ and $\omega(z)=$ $\frac{i}{2} g_{a \bar{b}} d z^{a} \wedge d \bar{z}^{\bar{b}}$ the Kähler form.

Note the close similarity of the $z$-independent prefactor with the prefactor of the Bousso-Polchinski distribution Eq. (15). Essentially this arises because the constraint Eq. (26) combined with the condition of supersymmetry roughly restricts the fluxes to be contained in a sphere of radius proportional to $\sqrt{L}$. As a result, we will get similarly huge numbers of actual IIb flux vacua in string theory — for the models analyzed in detail in $(28)$, we find $N_{\text {vac }}($ total $) \sim 10^{307}, 10^{393}, 10^{506}$.

\footnotetext{
${ }^{1}$ To be more precise, this distribution is obtained by dropping the absolute value signs around the Jacobian determinant generalizing $\left|V^{\prime \prime}(\phi)\right|$ in Eq. (18). As a result some vacua will be counted negatively, hence the density is an index density rather than an absolute density. This nevertheless gives a good estimate of the actual density, and in any case a lower bound. Absolute densities can be obtained as well, but are more complicated.
} 
The last of these figures is the justification for the often quoted number $10^{500}$ as the total number of string vacua. However, as discussed in detail in (32), there are many further uncertainties even in counting the known classes of vacua, so this famous number should not be taken too seriously. Furthermore, the number we are ultimately most interested in, namely that of vacua similar to our own, is even less well understood at present; it could still be the case that only a few vacua (or even none) fit all the constraints implicit in the existing data. The point rather is that the problem of computing numbers of vacua with specific properties is both mathematically well posed (at least, as much so as string/M theory itself), and far easier than constructing the actual vacua. Thus, we can expect steady progress in this direction, leading to results of fairly direct phenomenological interest, as we will shortly explain.

The distribution Eq. (27) has some interesting structure. It diverges (remaining integrable) when the curvature diverges, which happens near so-called conifold degenerations of the Calabi-Yau manifold $X$, corresponding to a 3 -sphere in $X$ collapsing to zero size. Near this 3-cycle, the Calabi-Yau can be described by an equation of the form $x_{1}^{2}+x_{2}^{2}+x_{3}^{2}+x_{4}^{2}=z$ in $\mathbb{C}^{4}$, with the 3 -cycle being the real slice through it and $z=0$ the conifold point. The vacuum density near this point is given by (52):

$$
d N_{\operatorname{vac}}(z) \sim \frac{d|z|}{|z| \log ^{2}|z|^{-2}} \sim d\left(\log |z|^{-1}\right)^{-1}
$$

Note this is approximately scale invariant, naturally allowing hierarchically small scales for $z$, similar to the toy model distribution Eq. (23). This is no coincidence: near the conifold point, there is a pair of periods $\Pi(z) \sim(z, z \log z-z)$, just like in our toy model.

In the case at hand, small values of $z$ give rise to large warped $A d S_{5}$-like 
throats in the compactification (25), which have a dual gauge theory description through the AdS-CFT correspondence (54). In terms of the dual gauge coupling $g$, the distribution is simply uniform $d N_{\text {vac }} \sim d g^{2}$. The enhancement of vacua at small $z$ may be of more than academic interest: such warped throats have many possible phenomenological uses. They can provide a natural embedding of the Randall-Sundrum scenario (55) in string theory; they give rise to natural models of warped (and hence exponentially low-scale) supersymmetry breaking (56); and some of the simplest inflationary scenarios in string theory make use of such throats (57).

From this one-parameter distribution one can reasonably guess that the majority of IIb flux vacua will contain such warped throats, by combining the fact that some sizable fraction contains such a throat in the 1-parameter case with the simple argument that the probability of having no such throats in an $n$-modulus case can be expected to be roughly the $n$-th power of the probability of having no such throats in the one modulus case, becoming small at large $n$. Some more concrete evidence for this has been given in (58).

Distributions of other quantities besides the moduli have also been obtained for IIb ensembles. For example the distribution of cosmological constants of supersymmetric vacua near zero turns out to be uniform: $d N_{\text {vac }}(\Lambda) \sim \theta(-\Lambda) d \Lambda \sim$ $d|W|^{2}$, and the same is true for the string coupling constant: $d N_{\mathrm{vac}}\left(g_{s}\right) \sim d g_{s}$. If the Kähler moduli are stabilized according to the KKLT scenario, the distribution of KK scales $M_{K K}$ is roughly given by $d N_{\text {vac }}\left(M_{K K}\right) \sim e^{-c M_{K K}^{-4}} d\left(M_{K K}^{-4}\right)$ with $c$ some constant depending on the compactification manifold. This shows that low KK scales, (i.e. large extra dimensions) are statistically excluded in this scenario.

Distributions for other ensembles such as M-theory or type IIa flux vacua vacua 
have been worked out as well, and studies of distributions of discrete D-brane data such as gauge groups and matter content of intersecting brane models have been initiated. We refer to (32) and references therein for more details.

\subsection{Supersymmetry breaking scale}

Perhaps most interesting from a phenomenological point of view is the scale of supersymmetry breaking. We would like to know, out of all the string/M theory vacua which agree with existing data, is it likely that we live in one with low scale breaking, leading to discovery of superpartners at LHC, or not?

Of course, to answer this question conclusively, one would need a much better handle on many issues, both affecting the distribution of vacua, and also how cosmological selection mechanisms and the like influence actual probabilities on

the string theory landscape. Since at present we know very little about the second question, we will stick to the first, and discuss the better defined number distributions of string flux vacua.

Let us say that if many more vacua have property " $X$ " than property "not $X$ ", then $X$ is "favored," at least within the considerations we discuss. Another term for this type of consideration is "stringy naturalness." Of course, if it were to turn out that the probabilities of different vacua were roughly equal, this would lead directly to a statistical prediction. Even if not, if the probabilities to obtain vacua were uncorrelated with the property of interest, one would also get a statistical prediction. On the other hand, a particular cosmological model might lead to probabilities which were actually correlated with the property of interest, requiring one to balance various competing effects. This is a very interesting possibility but one which requires a broader discussion than we can make here. 
Anyways, keeping this qualification in mind, let us proceed.

Thus, we would like to compute $d N_{\text {vac }}\left(M_{\text {susy }}\right)$ given the observed values of $\Lambda \sim 0$ and $M_{E W} \sim 100 \mathrm{GeV}$, with $M_{\text {susy }}^{4}$ defined to be equal to the positive definite contribution to the supergravity potential, i.e.

$$
M_{\text {susy }}^{4} \equiv \sum_{a}\left|F_{a}\right|^{2}+\sum_{A} D_{A}^{2}
$$

where $F_{i}=e^{K / 2} D_{i} W$. Suppose this followed an approximate power law distribution, $d N_{\text {vac }}\left(M_{\text {susy }}\right) \sim M_{\text {susy }}^{\alpha} d M_{\text {susy }}$, then for $\alpha<-1$ vacuum statistics would favor low scale susy, while for $\alpha>-1$ it would not.

For purposes of comparison, let us begin with the standard prediction of field theoretic naturalness, implicit in the motivation often given for low scale supersymmetry breaking based on the hierarchy problem. This is

$$
d N_{\text {vac }}^{F T}\left(M_{\text {susy }}\right) \sim\left(\frac{M_{E W}^{2} M_{\mathrm{Pl}}^{2}}{M_{\text {susy }}^{4}}\right)\left(\frac{\Lambda}{M_{\text {susy }}^{4}}\right) f\left(M_{\text {susy }}\right),
$$

where the first factor represents the electroweak scale tuning, and the second one the cosmological constant tuning assuming a supersymmetric vacuum has cosmological constant zero (as is the case in rigid supersymmetric field theory). The factor $f(M)$ represents the a priori distribution coming out of the underlying theory, independent of these tuning requirements. If we grant that this is set by strong gauge dynamics as in conventional field theory models of supersymmetry breaking, a reasonable ansatz might be $f(M)=d M / M$, analogous to Eq. (28). This would lead to $\alpha=-9$ and a clear statistical prediction.

On the other hand, this is leaving out all of the fluxes and hidden sectors which were postulated in the Bousso-Polchinski model, and are generic in actual string/M theory compactifications. Since the expression Eq. (29) is a sum of squares, a simple model for their effect is that $M_{\text {susy }}$ receives many independent 
positive contributions, about as many as there are fluxes or at least moduli, leading to prior distributions of the form $(\overline{59}, 60)$

$$
f\left(M_{\text {susy }}\right) \sim d M_{\text {susy }}^{4 K}
$$

This would lead to a large positive value for $\alpha$ and overwhelmingly prefer high energy supersymmetry breaking.

Thus, depending on our microscopic picture, we arrive at very different conclusions. However, both of these simple considerations have flaws. The simplest problem with Eq. (30) is the factor $\Lambda / M_{\text {susy }}^{4}$. Instead, cosmological constant distributions of flux vacua generally go as $\Lambda / M_{F}^{4}$, with $M_{F}=M_{K K}$ or $M_{F}=M_{P}$ or some other fundamental scale. In other words, the tuning problem of the cosmological constant is not helped by supersymmetry. Essentially the reason is that in supergravity, the effective potential receives both positive and negative contributions, with negative contributions $\sim-|W|^{2}$ persisting for supersymmetric vacua. In the case of flux vacua, these negative contributions are distributed roughly uniformly up to some fundamental scale $M_{F}^{4}$, independent of the supersymmetry breaking scale, as we saw at the end of section 3.3 . This leads to a tuning factor $\Lambda / M_{F}^{4}$ instead of $\Lambda / M_{\text {susy }}^{4}$ in Eq. (30), resulting in $\alpha=-5$, still favoring low scale supersymmetry, but rather less so.

There are many other implicit assumptions and even gaps in the arguments for Eq. (30), many already recognized in the literature. Among those which implicitly favor low scale breaking, the expression Eq. (30) assumes a generic solution to the $\mu$ problem, as well as to the other known problems of supersymmetric phenomenology such as FCNC's etc. As an example on the other side, there might exist some generic class of models in which the supersymmetric contributions to $W$ are forced to be small, say by postulating an $\mathrm{R}$ symmetry which is only broken 
along with supersymmetry breaking, restoring the $\Lambda / M_{\text {susy }}^{4}$ factor.

Let us however turn to the arguments leading to Eq. (31), which if true would potentially outweigh all of these other considerations. To examine this further, we need a microscopic model of supersymmetry breaking. In fact, one can expect a generic potential to contain many metastable supersymmetry breaking minima, not because of any "mechanism," but simply because generic functions have many minima. Indeed this was shown to be generic for IIb flux vacua in (53), leading to the distribution

$$
f\left(M_{\text {susy }}\right) \sim d\left(\frac{M_{\text {susy }}}{M_{F}}\right)^{12}
$$

in the regime $M_{\text {susy }} \ll M_{F}$. This would still favor high superymmetry breaking scales in Eq. (30), but much less so than Eq. (31) - the flaw in the argument for the latter is that the different contributions to $F^{2}=\sum_{a}\left|F_{a}\right|^{2}$ are not independent, but correlated by the critical point conditions $\partial_{a} V=0$.

Although the specific power 12 may be surprising at first, it has a simple explanation (61, 62). Let us consider a generic flux vacuum with $M_{\text {susy }} \ll M_{F}$. Since one needs a goldstino for spontaneous susy breaking, at least one chiral superfield must have a low mass; call it $\phi$. Generically, the flux potential gives order $M_{F}$ masses to all the other chiral superfields, so they can be ignored, and we can analyze the constraints in terms of an effective superpotential reduced to depend on the single field $\phi$,

$$
W(\phi)=W_{0}+a \phi+b \phi^{2}+c \phi^{3}+\ldots
$$

The conditions for a metastable supersymmetric vacuum are then $|a|=M_{\text {susy }}^{2}$, $|b|=2|a|$ (this follows from the equation $V^{\prime}=0$ ), and finally $|c| \sim|a|$ (as explained in (53) and many previous discussions, this is necessary so that $V^{\prime \prime}>0$. This also requires a lower bound on the curvature of the moduli space metric). 
Furthermore, an analysis of flux superpotentials along the lines of our previous discussion bears out the expectation that the parameters $(a, b, c)$ are independent and uniformly distributed complex parameters, up to the flux potential cutoff scale $M_{F}$. To get low scale breaking, all three complex parameters must be tuned to be small in magnitude, leading directly to Eq. (32).

The flaw in applying a standard naturalness argument here is very simple; one needs to tune several parameters in the microscopic theory to accomplish a single tuning at the low scale. Of course, if the underlying dynamics correlated these parameters, one could recover natural low scale breaking. Thus, we have not replaced the paradigm of naturalness, but rather sharpened and extended it. But even granting that theories with such dynamics exist, the question becomes whether among the many possibilities contained in the string theory landscape, the vacua realizing them are sufficiently numerous to dominate the simpler "fine tuning" scenario. After all, the fine tuning we are trying to explain is only of order $\left(100 \mathrm{GeV} / M_{P}\right)^{2} \sim 10^{-34}$, and for all we know the fraction of string theory vacua which do this by low scale supersymmetry breaking is even smaller. While it will probably be some time before we can convincingly answer such questions, thinking about them has already shed new light on many old problems.

Having a precise microscopic picture from string theory becomes particularly important when one is evaluating the naturalness of discrete choices, or trying to weigh the importance of competing effects. As an example, let us consider the possibility that some of the problems we discussed (such as large $|W|$ leading to $\Lambda / M_{F}^{4}$ ) could be solved by postulating a discrete $\mathrm{R}$ symmetry. Indeed, almost all existing proposals for natural models of low scale breaking, such as those discussed in (63), rely on this postulate. 
Unfortunately for such proposals, there is a simple argument that discrete $\mathrm{R}$ symmetry is heavily disfavored in flux vacua (64). First, a discrete symmetry which acts on Calabi-Yau moduli space, will have fixed points corresponding to particularly symmetric Calabi-Yau manifolds; at one of these, it acts as a discrete symmetry of the Calabi-Yau. Such a symmetry of the Calabi-Yau will also act on the fluxes, trivially on some and non-trivially others. To get a flux vacuum respecting the symmetry, one must turn on only invariant fluxes. Now, looking at examples, one finds that typically an order one fraction of the fluxes transform non-trivially; for definiteness let us say half of them. Thus, applying Eq. (27) and putting in some typical numbers for definiteness, we might estimate

$$
\frac{N_{\text {vac symmetric }}}{N_{\text {vac all }}} \sim \frac{L^{K / 2}}{L^{K}} \sim \frac{10^{100}}{10^{200}} .
$$

Thus, discrete symmetries of this type come with a huge penalty. While one can imagine discrete symmetries with other origins for which this argument might not apply, since $W$ receives flux contributions, it clearly applies to the $\mathrm{R}$ symmetry desired in branch (3), and probably leads to suppressions far outweighing the potential gains. (Fortunately, this does not apply to $\mathrm{R}$ parity).

This is in stark contrast to traditional naturalness considerations, in which all symmetries are "natural." And there are other examples of string vacuum distributions which come out differently from traditional expectations, or which are different among different classes of string/M theory vacua, as discussed in $(32,49)$ and references there.

Other distributions are simply not predicted at all by traditional naturalness arguments. A primary example is the distribution of gauge groups and charged matter content. Suppose we were to find evidence for a new strongly coupled gauge sector (perhaps responsible for supersymmetry breaking, perhaps not), but 
had very limited information about the matter spectrum, say a single resonance. What should we expect for the gauge group? Simple guesses might be $S U(2)$ (the smallest nonabelian group), or $S U(4)$ (following the pattern $1-2-3-\ldots$ ). On the other hand, a huge amount of theoretical work has been devoted to the proposition that $S U(N)$ gauge theory becomes simpler as $N$ becomes large; should we not give this intuition equal weight?

While the considerations we just cited are unconvincing, in fact string/M theory does predict a definite distribution of gauge theories and matter contents, which has been explored in numerous recent works, including $(65,66,67,68,69,70)$ and many more cited in the reviews. Besides bearing on the susy breaking scale, such results could be useful in guiding searches for exotic matter, in motivating other proposals for dark matter, and so on.

\section{Implications for the testability of string theory}

As of 2007, it seems fair to say that while string theory remains by far the best candidate we have for a complete theory of fundamental physics, there is still no compelling empirical evidence for or against the claim that the theory describes our universe.

Many different approaches have been proposed to look for such evidence. Perhaps the simplest, and certainly the best founded in the history of our subject, is to look for "exotic" or "signature" physics which is easily modelled by string theory, and not by other theories. There are many such phenomena, such as excitation states of the string (Regge recurrences). Each is associated with a new energy scale, for example the string scale $M_{s}$ for excited string states. As another example, in higher dimensional theories, once one reaches energies of order the 
higher dimensional Planck scale $M_{P}$, one can have black hole creation. Presumably, Planck mass black holes decay very rapidly and this process can be thought of as creating an unstable particle, however it has been argued that the resulting distribution of decay products will look very different from other interactions. And, the higher dimensional Planck scale can in principle be far lower than the four-dimensional Planck scale, perhaps low enough to make this observable (for a recent review, see (71)).

One can add the Kaluza-Klein scale $M_{K K}$ to the list; while logically speaking the observation of extra dimensions is not a direct test of string theory, clearly it would have an equally profound significance for fundamental physics. There are a number of other "exotic" phenomena of which one can say the same thing, such as the possibility of non-trivial four dimensional fixed point field theories, and phenomena related to "warping." Again, it is not hard to propose scenarios in which this new physics will not be seen until we reach a new energy scale $M_{E X}$. Thus, the larger problem is to decide whether any of these phenomena are relevant in our universe.

The most direct approach to testing the theory is to find a way to probe these energy scales. At present, phenomenological constaints on all of these new energy scales appear to be very weak, ranging from just above current collider bounds, around a few $\mathrm{TeV}$, all the way up to the GUT and four dimensional Planck scales $M \geq 10^{16} \mathrm{GeV}$. In most of this parameter space, the exotic phenomena may well be inaccessible in terrestrial experiments and irrelevant in almost all astrophysical processes. Thus, while string theory can offer experimentalists many exciting possibilities, there is little in the way of guarantees, nor any clear way for such searches to falsify the theory. 
Thus, a central question for string theorists is to better constrain these scales theoretically. Since parameters such as the size of the extra dimensions are moduli, the considerations we have discussed in our review are clearly very relevant for this, and there are already many interesting suggestions. For example, it appears that in KKLT IIb flux vacua with many moduli, large extra dimensions are disfavored. On the other hand, there is an alternate regime in IIb theory in which the structure of the effective potential requires large extra dimensions (72), and it appears that other constructions such as IIa and M theory flux vacua may statistically favor large extra dimensions $(40,73)$. Thus the picture at present is not very clear; furthermore it seems very likely a priori that considerations from early cosmology bear on this particular question; but it is reasonable to expect significant theoretical progress on this question.

Again, this progress is likely to lead to "statistical predictions," in the sense that even if most vacua are shown to have some property (say for sake of discussion, large extra dimensions), there will be exceptions. To make a perhaps evident comment on the value of this, while in the hypothetical situation we discuss one would not be able to say that "ruling out" large extra dimensions would falsify string theory, one would at least know that one had drastically narrowed down the possibilities, allowing one to go on to determine the most promising next avenues for potential tests. In this way, getting a picture of the landscape is useful and perhaps even necessary in guiding the search for conclusive tests.

Let us now turn to the question of testability if we do not see exotic physics. To be more precise, suppose that all observed physics can be well described by some $4 \mathrm{~d}$ effective field theory coupled to gravity. This is certainly true at present, and it might well turn out so for physics at 1 and even $10 \mathrm{TeV}$ as well. While this 
would seem a frustrating possibility, one can certainly still hope to make contact with string theory from such data. After all, we believe that string/M theory has a finite number of vacua $(\overline{74})$, and thus can lead to a finite number of $4 \mathrm{~d}$ low energy theories; could we imagine showing that the data is fit by none of these theories, thus falsifying the theory?

Approaches of this type include the following.

1. Find "no-go" arguments that certain phenomena, which can be described by effective field theory, in fact cannot arise in string theory. For example, one can argue this for time variation of the fine structure constant (75). One can also place bounds on gauge couplings (admittedly, far from the observed values) (76).

2. Similarly, we could try to use the phenomena to rule out competing theories, thereby getting circumstantial evidence for string theory. The basic example here is that, at present, there is no other generally accepted theory of four dimensional quantum gravity, and this is commonly taken as circumstantial evidence for string theory. Of course, one should not take this too seriously until it can be proven that alternatives do not exist. In our opinion, the most promising alternative to study at present is the idea that certain extended supergravity theories might provide finite theories of gravity (77, 78).

3. Perhaps physics at the few TeV scale of LHC and ILC will turn out to show some remarkable simplicity which can easily be reproduced by string theory compactification. One idea is to focus on properties of the particular GUT theories obtained by heterotic string compactification. More recently, it has been suggested that certain D-brane "quiver gauge theories" leading 
to Standard-like models are preferred $(\overline{81,82})$.

4. Make statements about the "likely" distribution of predictions among all vacua of string theory. Then, to the extent that what we see is "likely," we again get circumstantial evidence for string theory.

Again, (1-3) suffer from the general problem that the phenomena being discussed may not actually be properties of our universe, while (4) suffers from the problem that we might just live in an "unlikely" universe. Thus, one would probably need to combine information from all of these approaches to make progress.

A more optimistic version of (4) holds that a better understanding of early cosmology and whatever mechanism populates the many vacua of the theory, will lead to a strongly peaked probability distribution which selects one or a handful of the candidate vacua in a way amenable to calculation. The search for gauge invariant and well-defined inflationary measures has been a 20-year struggle; recent progress is summarized in the short review (79). We note that even should one find a natural and computable candidate measure, actually finding the preferred vacua may be a daunting problem. It is not hard to imagine scenarios in which this is impossible even in principle, because of fundamental limitations coming from the theory of computational complexity (80).

It is hard at present to judge the prospects for any of these approaches. From thinking about historical analogies, a tentative conclusion for the problem at hand is that while a great deal can be learned on the theoretical side, perhaps eventually allowing us to propose a definite test, ultimately convincing evidence for string theory will probably have to come from observing some sort of exotic physics. A natural place to look for this is early cosmology, as the physics of inflation involves very high energies, with $V \sim M_{G U T}^{4}$ in many models. Several of the 
proposed models of inflation in string theory have characteristic signatures which (if sufficiently well measured) encode stringy physics. These include networks of cosmic D and F strings formed during the exit from brane inflation $(83,84,85)$ and non-Gaussian signals in the CMB radiation which probe the specific nonlinearities of the DBI action $(86,87,88)$.

Space prohibits a detailed discussion of these and many other interesting ideas. We conclude by noting that while the present situation is not very satisfactory, there is every reason to be optimistic. In string/M theory, we have a theoretical framework which on the one hand is grounded in precise mathematics (so that many, even most theoretical suggestions can be falsified on internal grounds), yet which on the other hand shows significant promise of making contact with observable physics. There are many well-motivated directions for improving the situation, and good reasons to believe that substantial progress will be made in the future.

\section{Acknowledgements}

The work of M.R.D. was supported by DOE grant DE-FG02-96ER40959, and the work of S.K. was supported in part by a David and Lucile Packard Foundation Fellowship, the DOE under contract DE-AC02-76SF00515, and the NSF under grant number 0244728 .

Literature Cited

1. J. Scherk and J. H. Schwarz, Phys. Lett. B 52, 347 (1974).

2. J. H. Schwarz, Phys. Rept. 89, 223 (1982).

3. M. B. Green and J. H. Schwarz, Phys. Lett. B 149, 117 (1984). 
4. P. Candelas, G. T. Horowitz, A. Strominger and E. Witten, Nucl. Phys. B 258, 46 (1985).

5. M. Dine and N. Seiberg, Phys. Lett. B 162, 299 (1985).

6. A. Sen, Prepared for Les Houches Summer School: Session 76: Euro Summer School on Unity of Fundamental Physics: Gravity, Gauge Theory and Strings, Les Houches, France, 30 Jul - 31 Aug 2001

7. K. A. Intriligator and N. Seiberg, Nucl. Phys. Proc. Suppl. 45BC, 1 (1996) [arXiv:hep-th/9509066].

8. S. Weinberg, Rev. Mod. Phys. 61, 1 (1989).

9. S. Weinberg, arXiv:astro-ph/0005265.

10. M. S. Chanowitz, Ann. Rev. Nucl. Part. Sci. 38, 323 (1988).

11. E. G. Adelberger, B. R. Heckel and A. E. Nelson, Ann. Rev. Nucl. Part. Sci. 53, 77 (2003) [arXiv:hep-ph/0307284].

12. T. Banks, D. B. Kaplan and A. E. Nelson, Phys. Rev. D 49, 779 (1994) [arXiv:hep-ph/9308292].

13. B. de Carlos, J. A. Casas, F. Quevedo and E. Roulet, Phys. Lett. B 318, 447 (1993) [arXiv:hep-ph/9308325].

14. G. D. Coughlan, W. Fischler, E. W. Kolb, S. Raby and G. G. Ross, Phys. Lett. B 131, 59 (1983).

15. P. J. E. Peebles and B. Ratra, Rev. Mod. Phys. 75, 559 (2003) [arXiv:astro-ph/0207347].

16. T. Banks, Phys. Rev. Lett. 52, 1461 (1984).

17. A.D. Linde, Rept. Prog. Phys. 47, 925 (1984).

18. S. Weinberg, Phys. Rev. Lett. 59, 2607 (1987).

19. W. Lerche, D. Lust and A. N. Schellekens, Nucl. Phys. B 287, 477 (1987). 
20. R. Bousso and J. Polchinski, JHEP 0006, $006 \quad$ (2000)

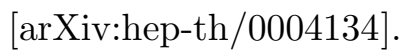

21. S. Gukov, C. Vafa and E. Witten, Nucl. Phys. B 608, 477 (2001) [arXiv:hep-th/9906070].

22. K. Dasgupta, G. Rajesh and S. Sethi, JHEP 9908, 023 (1999) [arXiv:hep-th/9908088].

23. P. Mayr, Nucl. Phys. B 593, 99 (2001) [arXiv:hep-th/0003198].

24. G. Curio, A. Klemm, D. Lust and S. Theisen, Nucl. Phys. B 609, 3 (2001) [arXiv:hep-th/0012213].

25. S.B. Giddings, S. Kachru and J. Polchinski, Phys. Rev. D 66, 106006 (2002) [arXiv:hep-th/0105097].

26. E. Silverstein, arXiv:hep-th/0106209.

27. S. Kachru, R. Kallosh, A. Linde and S. P. Trivedi, Phys. Rev. D 68, 046005 (2003) [arXiv:hep-th/0301240].

28. F. Denef, M. R. Douglas and B. Florea, JHEP 0406, 034 (2004) [arXiv:hep-th/0404257].

29. F. Denef, M. R. Douglas, B. Florea, A. Grassi and S. Kachru, arXiv:hep-th/0503124.

30. D. Lust, S. Reffert, E. Scheidegger, W. Schulgin and S. Stieberger, arXiv:hep-th/0609013.

31. L. Susskind, arXiv:hep-th/0302219.

32. M. R. Douglas and S. Kachru, arXiv:hep-th/0610102.

33. R. Blumenhagen, B. Kors, D. Lust and S. Stieberger, arXiv:hep-th/0610327.

34. M. Grana, Phys. Rept. 423, 91 (2006) [arXiv:hep-th/0509003].

35. E. Silverstein, arXiv:hep-th/0405068. 
36. A. R. Frey, arXiv:hep-th/0308156.

37. P. G. O. Freund and M. A. Rubin, Phys. Lett. B 97, 233 (1980).

38. J. McGreevy, E. Silverstein and D. Starr, arXiv:hep-th/0612121.

39. A. Saltman and E. Silverstein, JHEP 0601, 139 (2006) [arXiv:hep-th/0411271].

40. O. DeWolfe, A. Giryavets, S. Kachru and W. Taylor, JHEP 0507, 066 (2005) [arXiv:hep-th/0505160].

41. G. Villadoro and F. Zwirner, JHEP 0506, $047 \quad$ (2005) [arXiv:hep-th/0503169].

42. P.G. Camara, A. Font and L.E. Ibanez, JHEP 0509, 013 (2005) [arXiv:hep-th/0506066].

43. L. J. Dixon, J. A. Harvey, C. Vafa and E. Witten, Nucl. Phys. B 261, 678 (1985).

44. T. Banks and K. van den Broek, arXiv:hep-th/0611185.

45. M. Berkooz, M. R. Douglas and R. G. Leigh, Nucl. Phys. B 480, 265 (1996) [arXiv:hep-th/9606139].

46. R. Blumenhagen, M. Cvetic, P. Langacker and G. Shiu, Ann. Rev. Nucl. Part. Sci. 55, 71 (2005) [arXiv:hep-th/0502005].

47. K. Intriligator, N. Seiberg and D. Shih, JHEP 0604, 021 (2006) [arXiv:hep-th/0602239].

48. H. Ooguri and Y. Ookouchi, Phys. Lett. B 641, 323 (2006) [arXiv:hep-th/0607183].

49. J. Kumar, Int. J. Mod. Phys. A 21, 3441 (2006) [arXiv:hep-th/0601053].

50. M. R. Douglas, JHEP 0305, 046 (2003) [arXiv:hep-th/0303194].

51. S. Ashok and M. R. Douglas, JHEP 0401, 060 (2004) 
[arXiv:hep-th/0307049].

52. F. Denef and M. R. Douglas, JHEP 0405, $072 \quad$ (2004) [arXiv hep-th/0404116].

53. F. Denef and M. R. Douglas, JHEP 0503, 061 (2005) [arXiv:hep-th/0411183].

54. I. R. Klebanov and M. J. Strassler, JHEP 0008, 052 (2000) [arXiv:hep-th/0007191].

55. L. Randall and R. Sundrum, Phys. Rev. Lett. 83, 3370 (1999) [arXiv:hep-ph/9905221].

56. S. Kachru, J. Pearson and H. L. Verlinde, JHEP 0206, 021 (2002) [arXiv:hep-th/0112197].

57. S. Kachru, R. Kallosh, A. Linde, J. M. Maldacena, L. McAllister and S. P. Trivedi, JCAP 0310, 013 (2003) [arXiv:hep-th/0308055].

58. A. Hebecker and J. March-Russell, arXiv:hep-th/0607120.

59. M. R. Douglas, arXiv:hep-th/0405279.

60. L. Susskind, arXiv:hep-th/0405189.

61. M. Dine, D. O’Neil and Z. Sun, JHEP 0507, 014 (2005) [arXiv:hep-th/0501214].

62. G. F. Giudice and R. Rattazzi, Nucl. Phys. B 757, 19 (2006) [arXiv:hep-ph/0606105].

63. M. Dine, J. L. Feng and E. Silverstein, Phys. Rev. D 74, 095012 (2006) [arXiv:hep-th/0608159].

64. M. Dine and Z. Sun, JHEP 0601, 129 (2006) [arXiv:hep-th/0506246].

65. R. Blumenhagen, F. Gmeiner, G. Honecker, D. Lust and T. Weigand, Nucl. Phys. B 713, 83 (2005) [arXiv:hep-th/0411173]. 
66. T. P. T. Dijkstra, L. R. Huiszoon and A. N. Schellekens, Nucl. Phys. B 710, 3 (2005) [arXiv:hep-th/0411129].

67. F. Gmeiner, Fortsch. Phys. 54, 391 (2006) [arXiv:hep-th/0512190].

68. M. R. Douglas and W. Taylor, arXiv:hep-th/0606109.

69. J. Kumar and J. D. Wells, JHEP 0509, 067 (2005) [arXiv hep-th/0506252].

70. K. R. Dienes, Phys. Rev. D 73, 106010 (2006) [arXiv:hep-th/0602286].

71. G. Landsberg, J. Phys. G 32, R337 (2006) [arXiv:hep-ph/0607297].

72. V. Balasubramanian, P. Berglund, J. P. Conlon and F. Quevedo, JHEP 0503, 007 (2005) [arXiv:hep-th/0502058].

73. B. S. Acharya, F. Denef and R. Valandro, JHEP 0506, 056 (2005) [arXiv:hep-th/0502060].

74. B. S. Acharya and M. R. Douglas, arXiv:hep-th/0606212.

75. T. Banks, M. Dine and M. R. Douglas, Phys. Rev. Lett. 88, 131301 (2002) [arXiv:hep-ph/0112059].

76. N. Arkani-Hamed, L. Motl, A. Nicolis and C. Vafa, arXiv:hep-th/0601001.

77. Z. Bern, L. J. Dixon and R. Roiban, arXiv:hep-th/0611086

78. M. B. Green, J. G. Russo and P. Vanhove, arXiv:hep-th/0611273

79. A. Vilenkin, arXiv:hep-th/0609193.

80. F. Denef and M. R. Douglas, arXiv:hep-th/0602072.

81. D. Berenstein and S. Pinansky, arXiv:hep-th/0610104.

82. H. Verlinde and M. Wijnholt, arXiv:hep-th/0508089.

83. S. Sarangi and S. H. H. Tye, Phys. Lett. B 536, 185 (2002) [arXiv:hep-th/0204074].

84. E. J. Copeland, R. C. Myers and J. Polchinski, JHEP 0406, 013 (2004) [arXiv:hep-th/0312067]. 
85. G. Dvali and A. Vilenkin, JCAP 0403, 010 (2004) [arXiv:hep-th/0312007].

86. M. Alishahiha, E. Silverstein and D. Tong, Phys. Rev. D 70, 123505 (2004) [arXiv:hep-th/0404084].

87. D. Babich, P. Creminelli and M. Zaldarriaga, JCAP 0408, 009 (2004) [arXiv:astro-ph/0405356].

88. X. Chen, M. x. Huang, S. Kachru and G. Shiu, arXiv:hep-th/0605045. 